Una M. Röhr-Sendlmeier und Jenny Yun

\title{
Familienvorstellungen im Kulturkontakt: ein Vergleich italienischer, türkischer, koreanischer und deutscher junger Erwachsener in Deutschland
}

Family concepts of young adults in Germany: A comparison of Italian, Turkish and Korean migrants and their German peers

\begin{abstract}
Zusammenfassung
In zwei Studien mit 215 Personen wurden die Vorstellungen zu Partnerschaft, Ehe und Familie von italienischen, türkischen und koreanischen jungen Erwachsenen der zweiten Migrantengeneration mit denen deutscher junger Erwachsener verglichen. In einer ersten Studie mit 115 Befragten der vier Ethnien, die repräsentativ nach dem Bildungsniveau zusammengesetzt waren, erwiesen sich die Voraussetzungen für eine Familiengründung und die antizipierte Rollenverteilung in der Familie als sehr unterschiedlich. So zeigten die italienischen und türkischen Erwachsenen eine starke Orientierung an familiären Traditionen und Werten, während die koreanischen und deutschen Teilnehmer die Individualität und die Rationalität des Einzelnen in den Vordergrund stellten. In einer zweiten Studie wurden weitere 100 Personen rekrutiert, um die Vorstellungen junger Erwachsener derselben ethnischen Gruppen mit Gymnasialbildung zu erhellen. Die Familienvorstellungen der jungen ausländischen Erwachsenen mit Abitur ähnelten denen der deutschen Untersuchungsteilnehmer. Vor allem italienische und türkische Frauen mit Gymnasialbildung zeigten signifikant andere Zukunftsvorstellungen als die entsprechenden Gruppen mit gemischten Bildungshintergründen.
\end{abstract}

Schlagworte: Migration, zweite Generation, Assimilation, Familienvorstellungen, Bildungsniveau, Geschlecht

\section{Abstract}

Concepts of partnership, marriage and the family of a total of 215 young adults of different cultural backgrounds in Germany are compared. In an initial study, 115 German and Italian, Turkish and Korean young adults of the second migrant generation were interviewed; their achieved educational levels were representative for each group. The prerequisites for establishing a family and the anticipated role allocation within the family turned out to be very different. The Italian und Turkish adults showed a strong orientation towards family traditions and values, whereas the Korean and German participants emphasized individuality and rationality of the individual. In a second complementary study, 100 young adults of the same ethnic groups, who now all had an educational level of comprehensive secondary school, were recruited to investigate the family concepts in these specific groups. The young migrants had developed similar family concepts to those of their German peers. Especially Italian and Turkish women with comprehensive secondary school education revealed significantly different perceptions of the future in comparison to the concepts of the respective 
groups with mixed educational back- Key words: migration, second generation, grounds.

assimilation, family concepts, educational level, gender

\section{$1 \quad$ Einleitung}

Generationsbeziehungen sind für das Verständnis von Familien ausländischer Herkunft aus zwei Gründen von besonderer Bedeutung: (1) Die meisten Eltern sind Arbeitsmigranten, die Mitte der 1960er Jahre als angeworbene Arbeitskräfte ursprünglich für einen zeitlich begrenzten Aufenthalt nach Deutschland kamen (Lajios 1998). Sie stammen aus Gesellschaften ohne ausgebautes System sozialstaatlicher Sicherung, in denen Absicherungen gegen die Risiken des Lebens zum größten Teil zwischen den Generationen innerhalb der Familien erbracht werden. (2) Die Migrationssituation selbst hat unmittelbare Auswirkungen auf die Generationsbeziehungen, lassen sich doch viele Migrationsziele nur im Generationenzusammenhang legitimieren und realisieren. In Migrantenfamilien sind intergenerative Beziehungen besonders hoch motiviert und stärker koordiniert, als dies in nichtgewanderten Familien in der Herkunfts- oder in der Aufnahmegesellschaft der Fall ist (Nauck/Niephaus 2001; Nauck 2002). Welche Vorstellungen von Partnerschaft, Ehe und der Gründung einer eigenen Familie haben junge Erwachsene in Deutschland vor unterschiedlichen Migrationshintergründen entwickelt?

Bisherige Studien beleuchten die Situation einzelner Migrantengruppen in Deutschland und Facetten wie die Eltern-Kind-Beziehung, die Partnerschaft oder die Bildungsmobilität (vgl. z.B. Seifert 2000; Straßburger 2000; Trommsdorff 2001; Vetter 2001; Nauck 2002; Gültekin 2003). Ein multivariater Zugang stellt die Ausnahme dar (etwa Weidacher 2000). Als moderierende Faktoren der Akkulturation sind nach dem Stressbewältigungsansatz von Berry (1997) das Alter, das Geschlecht, die Erziehung, die Migrationsmotivation, Erfahrungen in der Migrationssituation und die kulturelle Distanz etwa in Sprache und Religion sowie Faktoren der Persönlichkeit anzusehen. Was ist zu diesen Faktoren bislang bekannt?

Die Bildungssituation der zweiten Generation hat sich im Vergleich zu der ihrer Eltern deutlich verbessert. Auch wenn weiterhin große Defizite insbesondere auf Seiten italienischer und türkischer Schülerinnen und Schüler bestehen (RöhrSendlmeier 1992; Bürkner 1998; Schönpflug 2003), durchläuft inzwischen ein beachtlicher Teil von ihnen erfolgreich die Realschule (Mitteilung der Beauftragten der Bundesregierung für die Belange der Ausländer 1997; Seifert 2000). Zwischen 1975 und 1997 stieg die Zahl der Studierenden türkischer Herkunft an deutschen Hochschulen von etwa 4.000 auf rund 21.000 (Schulze/Soja 2003). Die Identitätsentwicklung ausländischer Jugendlicher wird von der Familie und der Gruppe der Gleichaltrigen nicht immer in dieselbe Richtung gelenkt. Die Jugendlichen wollen nicht die Normen und Werte ihrer Eltern unreflektiert zum Inhalt ihres Lebens machen; sie wollen aber auch nicht die Ideale der Aufnahmegesellschaft unhinterfragt annehmen (Röhr-Sendlmeier 1990; Bratic/Viehböck 1994; Lajios 1998). Genau wie die deutschen Gleichaltrigen stehen die Migranten im jungen Erwachsenenal- 
ter vor Aufgaben wie Partnersuche, Familien- und Existenzgründung (Peukert 1996; Krampen/Reichle 2002). In zwei vom Bundesministerium für Arbeit und Sozialordnung in Auftrag gegebene Repräsentativerhebungen $(1986,1996)$ wurden ausländische Eltern gefragt, ob sie damit einverstanden wären, wenn ihr Kind einen Deutschen oder eine Deutsche heiratete. 1995 bejahten mehr als 50\% der türkischen (1985: 33\%) und rund 90\% der italienischen Eltern (1985: 65\%) diese Frage. Die Akzeptanz interethnischer Ehen hat bei den befragten Gruppen nach diesen Zahlen stark zugenommen (vgl. Bundesministerium für Familie, Senioren, Frauen und Jugend 1997). Dagegen fand Vetter (2001), dass türkische Eltern zu 66,5\% ,wahrscheinlich nicht“ oder ,,auf keinen Fall“ zustimmen würden, wenn ihr Kind einen deutschen Partner wählte; nur 4,5\% würden dies ,auf jeden Fall“ tolerieren. Nach dem 6. Familienbericht des Bundesministeriums für Familie, Senioren, Frauen und Jugend (2000) kommen binationale Partnerschaften besonders häufig vor, wenn mindestens ein Partner das Abitur oder die Fachhochschulreife besitzt.

Ungefähr ein Drittel der verheirateten jungen Italiener in Deutschland ist mit einem Ehepartner verheiratet, der kein Italiener ist. Der Anteil national gemischter Partnerschaften ist noch höher, wenn außereheliche Formen der Partnerschaft betrachtet werden, aber ein Viertel der jungen Menschen italienischer Herkunft lehnt eine Partnerschaft mit einem oder einer Deutschen völlig ab (Weidacher 2000). Italienische Migranten zeigen einen starken Zusammenhalt der Familie und eine enge Verbundenheit mit der katholischen Kirche (vgl. Loriedo 1996). Generationsbeziehungen sind als emotionale Beziehungen organisiert, bei denen ein intergenerativer Transfer von Dienstleistungen, Geld und Gütern zwar vorhanden ist, diese jedoch nicht die Beziehung definieren (Nauck 2000). Italiener gelten bei Deutschen als nahe stehende Europäer mit großen kulturellen Ähnlichkeiten (Thränhardt 1998) und als soziokulturell gut integriert (BoosNünning/Karakaşoğlu 2005). Berry (1997) verweist auf den Zusammenhang zwischen unproblematischen Akkulturationserfahrungen und als unproblematisch empfundenen Verhaltensveränderungen im Leben der Migranten. Durch die große Anzahl der Landsleute, die ebenfalls in Deutschland ansässig wurden, waren für italienische und türkische Arbeitsmigranten auch Alternativen zur Assimilation an die deutschen Lebensverhältnisse gegeben. Die Akzeptanz der Aufnahmegesellschaft gegenüber den Migrantengruppen hängt $u$. a. auch von der ethnischen Dichte der Minorität ab: Je höher die Dichte in bestimmten Regionen und je größer die Minoritätengruppe ist, umso geringer ist die Wahrscheinlichkeit der Akzeptanz durch die Aufnahmegesellschaft (Schönpflug 2003).

Den türkischen Migranten wurde die Assimilation noch zusätzlich erschwert, wenn sie aufgrund der Ausübung der islamischen Religion andere als der deutschen Bevölkerung bekannte Lebensweisen im Alltag praktizierten (Özkara 1990; Zentrum für Türkeistudien 1994). Die Bindung an den islamischen Glauben ist bei den türkischen Familien in Deutschland aber sehr unterschiedlich (Atabay 1998) und es besteht eine breite Variation an Werten und Normen sowohl in der Elterngeneration als auch bei den Kindern (Merkens 1997). Insbesondere türkischen Frauen wird noch immer die größte Fremdheit gegenüber gesellschaftlichen Normen und Werten in Deutschland nachgesagt und trotz ihrer offensiven Haltung zur 
Wanderung wurden sie in der Migrationsforschung bis in die 1990er Jahre hinein als eigenständig Handelnde weitgehend ignoriert (Gültekin 2003). In der Wahl der partnerschaftlichen Lebensformen verhalten sich türkische Frauen allerdings in der Tat häufig traditionell: Sie leben im Alter zwischen 21 und 25 Jahren bereits vielfach in ehelichen Gemeinschaften (vgl. Weidacher 2000; Münchmeier 2000; Fritzsche/Münchmeier 2000). Etwa 44\% der jungen türkischen Erwachsenen haben einen Partner oder eine Partnerin anderer, meist deutscher Nationalität. Bei deutschtürkischen Ehen muss jedoch bedacht werden, dass zunehmend Partner derselben Herkunft unterschiedliche Pässe haben. Tatsächlich kulturell gemischte Partnerschaften sind häufiger, wenn die Partner nicht-ehelich oder gar nicht zusammenleben. Fast 50\% der jungen Türken lehnen eine Partnerschaft mit einem oder einer Deutschen ab (Straßburger 2000; Weidacher 2000).

Die Situation der südkoreanischen Arbeitsmigranten unterscheidet sich von der der italienischen und türkischen Familien in Deutschland. Ihre Anzahl ist deutlich kleiner. Vor allem die südkoreanischen Frauen, die als Krankenschwestern nach Deutschland kamen, wurden von ihrer eigenen Regierung geschickt, um ihre Qualifizierung zu verbessern oder wurden von kirchlichen Organisationen angeworben. Die Koreaner in Deutschland gehören zu 50-60\% dem christlichen Glauben an (vgl. Yoo 1996). Das Bildungsniveau der meisten eingewanderten Koreanerinnen und Koreaner ist im Vergleich zu dem vieler anderer Migranten höher (Lee 1991). In asiatischen Gesellschaften sind Eltern-Kind-Beziehungen und damit zusammenhängende Entwicklungsaufgaben traditionell über die Lebensspanne hinweg klar strukturiert und eine stabile Eltern-Kind-Beziehung über die Lebensspanne ist selbstverständlich. Dabei gilt als hoher Wert die Kontinuität einer harmonischen Eltern-Kind-Beziehung mit klaren wechselseitigen Verpflichtungen: auf der einen Seite die elterliche Fürsorge, auf der anderen Seite die Loyalität und der Gehorsam der Kinder (vgl. z.B. Oerter/Oerter 1995; Trommsdorff 2001). In Deutschland konnten viele der traditionellen Normen und Werte nicht aufrechterhalten werden, auch aufgrund der Tatsache, dass die Frauen häufig den angeseheneren Beruf ausüben und mehr Geld verdienen. Die Kinder der koreanischen Migranten entwickeln dadurch, dass sie die deutsche Sprache lernen und zum Teil besser beherrschen als ihre Eltern, ein selbstbewussteres und kritischeres Verhalten, als es nach koreanischer Tradition erwünscht ist (vgl. Schönpflug 2003). Nach wie vor ist eine starke Leistungsorientierung innerhalb der koreanischen Familien zu erkennen. Über die Häufigkeiten und Art der interkulturellen Partnerschaften ist wenig bekannt (Lee 1990).

Sind vor dem skizzierten Hintergrund die Familienvorstellungen von italienischen, türkischen und koreanischen jungen Frauen und Männern denen deutscher Gleichaltriger ähnlich oder sind sie stärker von den Lebensformen und Überzeugungen ihrer Eltern beeinflusst? Hierzu sollen die folgenden Studien einige Antworten geben. 


\section{Studie 1: Familienvorstellungen von jungen Erwachsenen mit gemischten Bildungshintergründen}

\subsection{Methode}

Für die Zusammenstellung der Stichprobe mit ca. 30 Teilnehmern je Ethnie wurden folgende Prämissen festgelegt:

- Die Probanden sollten zwischen 20 und 30 Jahren alt sein, weil die Lebensspanne des jungen Erwachsenenalters durch Prozesse des Herausfindens, Erkennens und der Anpassung gekennzeichnet ist (z.B. Krampen/Reichle 2002).

- Die ausländischen Probanden sollten der zweiten Generation angehören und seit ihrer Geburt oder ihrer frühen Kindheit (1. bis 3. Lebensjahr) in Deutschland leben.

- Sie sollten aus einem Elternhaus kommen, in dem beide Elternteile aus demselben Land stammen, damit davon ausgegangen werden kann, dass ihre Entwicklung zu Hause vor allem durch ihre Herkunftskultur und außerhalb der Familie vor allem von der deutschen Umwelt geprägt wurde. Bei den deutschen Befragten sollten systematische Einflüsse durch den Kontakt mit Mitgliedern anderer kultureller Gruppen ausgeschlossen sein.

- Da sich häufig der erreichte Bildungsstand der jungen ausländischen von dem der deutschen Erwachsenen unterscheidet und die Partnerwahl beeinflusst, waren die einzelnen Bevölkerungsgruppen nach ihrem Bildungsstand repräsentativ für die jeweilige Bevölkerungsgruppe in Nordrhein-Westfalen zusammenzusetzen.

Die Daten des Landesamtes für Datenverarbeitung und Statistik NRW über die Schülerzahlen der Abschlussklassen im Schuljahr 1995/96 dienten als Orientierung zur Zusammenstellung der Stichprobe. Tabelle 1 zeigt, dass die angestrebte Repräsentativität - ausgedrückt durch den Zielwert - weitgehend erreicht wurde, und enthält Angaben zur Geschlechterverteilung der Probanden. 115 junge Frauen und Männer, die die genannten Prämissen erfüllten, konnten im Bereich Bonn, Köln und Leverkusen befragt werden. Alle italienischen Untersuchungsteilnehmer waren katholisch, alle türkischen gehörten dem Islam an. Von den deutschen Probanden waren 14, von den Koreanern 10 katholisch, 9 bzw. 17 waren Protestanten, 5 bzw. 2 waren konfessionslos.

Struktur und Aufbau des verwendeten Fragebogens orientierten sich zum einen an der 13. Shell Studie mit dem Thema ,Jugend 2000“, zum anderen an einer Studie zu den verschiedenen Ansichten von deutschen, koreanischen und amerikanischen Personen zur Elternschaft (vgl. Böttcher 1998) sowie an einem zusammenfassenden Bericht über familiale Erziehungsstile und die Sozialisation türkischer Kinder in Deutschland (vgl. Merkens/Schmidt 1997). 
Tabelle 1: Zusammensetzung der Stichprobe in Studie 1 nach Schulbesuch und Geschlecht

\begin{tabular}{|c|c|c|c|c|c|c|c|c|c|c|c|c|c|}
\hline & \multicolumn{3}{|c|}{ Hauptschule } & \multicolumn{3}{|c|}{ Realschule } & \multicolumn{3}{|c|}{ Gesamtschule } & \multicolumn{3}{|c|}{ Gymnasium } & \multirow{2}{*}{$\begin{array}{c}\text { Gesamt N } \\
(\mathrm{m} / \mathrm{w})\end{array}$} \\
\hline & & wert & $\begin{array}{c}\text { tat- } \\
\text { sächl. } \\
\mathrm{N}\end{array}$ & Zie & yert & $\begin{array}{c}\text { tat- } \\
\text { sächl. } \\
\mathrm{N}\end{array}$ & & vert & $\begin{array}{c}\text { tat- } \\
\text { sächl. } \\
\mathrm{N}\end{array}$ & & vert & $\begin{array}{c}\text { tat- } \\
\text { sächl. } \\
\mathrm{N}\end{array}$ & \\
\hline & $\%$ & $\mathrm{~N}$ & & $\%$ & $\mathrm{~N}$ & & $\%$ & $\mathrm{~N}$ & & $\%$ & $\mathrm{~N}$ & & \\
\hline $\begin{array}{l}\text { Deut- } \\
\text { sche }\end{array}$ & 25 & 7 & 6 & 27 & 8 & 8 & 17 & 5 & 4 & 31 & 10 & 10 & $\begin{array}{c}28 \\
(13 / 15) \\
\end{array}$ \\
\hline Italiener & 50 & 15 & 15 & 21 & 6 & 6 & 19 & 6 & 5 & 10 & 3 & 3 & $\begin{array}{c}29 \\
(14 / 15) \\
\end{array}$ \\
\hline Koreaner & 1 & 0 & 0 & 17 & 5 & 2 & 9 & 3 & 1 & 73 & 22 & 26 & $\begin{array}{c}29 \\
(13 / 16) \\
\end{array}$ \\
\hline Türken & 50 & 15 & 15 & 14 & 4 & 3 & 28 & 9 & 9 & 8 & 2 & 2 & $\begin{array}{c}29 \\
(15 / 14)\end{array}$ \\
\hline Summe & & & 36 & & & 19 & & & 19 & & & 41 & $\begin{array}{c}115 \\
(55 / 60) \\
\end{array}$ \\
\hline
\end{tabular}

Ferner sind neue Fragestellungen und Antwortkategorien in den Fragebogen eingegangen. Probleme in Bezug auf den kulturübergreifenden Einsatz des Fragebogens (vgl. Horn 2003) traten nicht auf. Der Fragebogen mit 140 Items setzt sich aus fünf Teilbereichen zusammen: soziographischen Daten, Vorstellungen zur Partnerschaft, Vorstellungen zur Ehe, Angaben zur Herkunftsfamilie und Vorstellungen zur Familie, die man selber gründen möchte. Es wurden sowohl offene als auch geschlossene Frageformen verwendet. Unterkategorien der geschlossenen Fragen waren Mehrfachantworten, 5-stufige Likert-Skalen ( $1=$ sehr hohe Ablehnung bis $5=$ sehr hohe Zustimmung) und einfache Antworten. Das Ausfüllen des Fragebogens fand im Rahmen persönlicher Interviews statt, so dass bei Unklarheiten ein Ansprechpartner bereit stand.

Die gewonnenen Daten wurden sowohl deskriptiv, bivariat als auch multivariat untersucht. Die in varianzanalytischen Berechnungen als signifikant $(\mathrm{p}<0.05)$ für die individuellen Vorstellungen ausgewiesenen Variablen wurden dahingehend überprüft, ob sie sich auch bei multivariater Betrachtung in logistischen Regressionsanalysen als entscheidende Faktoren durchsetzen konnten. Nur solche Befunde werden im Ergebnisteil detaillierter aufgeführt. Als mögliche Einflussfaktoren wurden mit Rückgriff auf den Stand der Forschung die soziographischen Variablen „Ethnie“, „Schulbesuch“, „Geschlecht“ und „Religion“ ausgewählt. Um noch klarere Profile der ethnischen Gruppen zu erhalten, wurde anschließend in einer explorativen, orthogonalen Faktorenanalyse überprüft, inwiefern die einzelnen Fragebogen-Items als statistisch zusammengehörig zu betrachten sind. War die Ladung der einzelnen Items $\geq 0.4$, wurden sie in die Betrachtung eingeschlossen. Mit Hilfe von sechs neu gewonnenen Kategorien wurden für alle Probanden die Mittelwerte dieser Faktoren berechnet und über einfaktorielle Varianzanalysen signifikante Unterschiede in den Bewertungen der einzelnen ethnischen Gruppen gesucht. Abschließend wurde der Einfluss des Geschlechtes auf die Bewertung der einzelnen Kategorien überprüft. 


\subsection{Ergebnisse}

\subsubsection{Vorstellungen zur Partnerschaft}

$\mathrm{Zu}$ einer Partnerschaft mit deutschem Partner zeigten sich die koreanischen Befragten $(M=3.79)$ bereit, während sich die türkischen $(M=3.03)$ und italienischen $(\mathrm{M}=2.93)$ Teilnehmer eher neutral äußerten: In der Regressionsanalyse $(\mathrm{F}(2,84)=3.56, \mathrm{p}=0.033)$ wird die Zugehörigkeit als „Italiener“ $(\mathrm{T}=2.44, \mathrm{p}=$ $0.017)$ oder als „Türke“ $(\mathrm{T}=2.15, \mathrm{p}=0.034)$ als Prädiktor ausgewiesen. Bei der Bewertung der „Voraussetzungen für eine gute Partnerschaft“ schätzten alle Teilnehmer die Items ähnlich ein. Lediglich bei dem Item „gleiche Religion“ $(\mathrm{F}(4,110)=5.87, \mathrm{p}<0.001)$ wurde die Zugehörigkeit als ,Deutscher“ $(\mathrm{T}=4.28, \mathrm{p}$ $<0.001)(\mathrm{M}=1.76)$, „Italiener“ $(\mathrm{T}=2.11, \mathrm{p}=0.037)(\mathrm{M}=2.52)$ und „Koreaner“ $(\mathrm{T}=3.14, \mathrm{p}=0.002)(\mathrm{M}=2.17)$ als Prädiktor ausgewiesen; nur den türkischen Probanden war die gemeinsame Religion als Voraussetzung für die Partnerschaft wichtig $(M=3.24)$. $\mathrm{Zu}$ den ,Erwartungen an eine gute Partnerschaft“" wurden bei fünf Variablen signifikant unterschiedliche Bewertungen vorgenommen (siehe Tabelle 2). Als Prädiktor dominiert die ethnische Zugehörigkeit, auch wird das Geschlecht bedeutsam.

Tabelle 2: $\quad$ Signifikante Ergebnisse der Regressionsanalyse für den Bereich „Erwartungen an eine gute Partnerschaft“ in Studie $1(\mathrm{~N}=115)$

\begin{tabular}{|c|c|c|c|}
\hline Item & Beeinflussende Faktoren & $\mathrm{T}$ & $p$ \\
\hline $\begin{array}{l}\text { Je länger eine Beziehung andauert, desto } \\
\text { wahrscheinlicher wird eine Hochzeit. }\end{array}$ & $\begin{array}{l}\text { Deutsche: } M=2.50 \\
\text { (Koreaner } M=3.34 \text {; Italiener } \\
M=3.72 ; \text { Türken } M=3.82 \text { ) }\end{array}$ & 4.28 & $<0.001$ \\
\hline $\begin{array}{l}\text { In einer Beziehung sollte jeder ein gewis- } \\
\text { ses Maß an Freiraum für sich behalten. }\end{array}$ & $\begin{array}{l}\text { Italiener: } \mathrm{M}=3.97 \\
\text { (Türken } \mathrm{M}=4.41 \text {; Deutsche } \\
\mathrm{M}=4.57 ; \text { Koreaner } \mathrm{M}=4.62 \text { ) }\end{array}$ & 3.90 & $<0.001$ \\
\hline $\begin{array}{l}\text { Ich fühle mich meinem Partner zu Ehrlich- } \\
\text { keit und Treue verpflichtet. }\end{array}$ & $\begin{array}{l}\text { Italiener: } M=4.90 \\
\text { Türken: } M=4.82 \\
\text { (Koreaner } M=4.52 ; \text { Deutsche } \\
M=4.61 \text { ) }\end{array}$ & $\begin{array}{l}-2.98 \\
-2.97\end{array}$ & $\begin{array}{l}0.004 \\
0.004\end{array}$ \\
\hline $\begin{array}{l}\text { Mich stört es nicht, wenn mein Partner mal } \\
\text { ohne mich ausgeht. }\end{array}$ & $\begin{array}{l}\text { Deutsche: } \mathrm{M}=4.67 \\
\text { (Italiener } \mathrm{M}=3.48 \text {; Türken } \mathrm{M}=3.83 \text {; } \\
\text { Koreaner } \mathrm{M}=3.97 \text { ) }\end{array}$ & -3.70 & $<0.001$ \\
\hline $\begin{array}{l}\text { Ich finde es gut, wenn die Frau ihren Part- } \\
\text { ner mal zum Essen einlädt. }\end{array}$ & $\begin{array}{l}\text { Koreaner: } M=4.17 \\
\text { (Türken } M=4.48 \text {; Italiener } M=4.52 \text {; } \\
\text { Deutsche } M=4.78 \text { ) } \\
\text { weiblich: } M=4.62 \\
\text { (männlich } M=4.35 \text { ) }\end{array}$ & -2.34 & 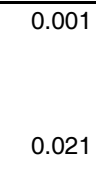 \\
\hline
\end{tabular}

Die beeinflussenden Faktoren wurden von der Regressionsanalyse als signifikant ausgewiesen; die in Klammern gesetzten Daten sind zur Information ergänzt ( 1 = sehr hohe Ablehnung bis 5 = sehr hohe Zustimmung).

\subsubsection{Vorstellungen zur Ehe}

Die prinzipielle Bereitschaft zu einer bikulturellen Ehe war bei allen Befragten gegeben, unabhängig von der Ethnie. Zur Bedeutung und den Werten der Ehe lassen 
sich dagegen unterschiedliche Bewertungen finden. Tabelle 3 enthält die ermittelten Ergebnisse. Neben der ethnischen Zugehörigkeit erwiesen sich die Schulbildung, die Religion und das Geschlecht als einflussreich. Auf die Frage, welche Voraussetzungen im Hinblick auf eine bikulturellen Ehe gegeben sein sollten, nannten die italienischen Untersuchungsteilnehmer bevorzugt eine ,ähnliche Kultur des Partners“, die koreanischen ,das Einverständnis der Eltern“ und die türkischen ,dieselbe Religion“ und „das Einverständnis der Eltern“.

Tabelle 3: $\quad$ Signifikante Ergebnisse der Regressionsanalyse für den Bereich „Bedeutung und Wert von Ehe“ in Studie $1(\mathrm{~N}=115)$

\begin{tabular}{|c|c|c|c|}
\hline Item & Beeinflussende Faktoren & $\mathrm{T}$ & $p$ \\
\hline Ehe ist ein Symbol ewiger Treue. & $\begin{array}{l}\text { Deutsche: } M=3.54 \\
\text { (Koreaner } M=4.00 \text {; Italiener } \\
M=4.44 ; \text { Türken } M=4.48 \text { ) }\end{array}$ & 2.88 & 0.005 \\
\hline $\begin{array}{l}\text { Ehe bedeutet Sicherheit und Geborgen- } \\
\text { heit. }\end{array}$ & $\begin{array}{l}\text { Christen: } \mathrm{M}=3.87 \\
\text { (Nicht-Christen } \mathrm{M}=4.36 \text { ) }\end{array}$ & 2.10 & 0.038 \\
\hline $\begin{array}{l}\text { Die Ehe verbessert die Beziehung der bei- } \\
\text { den Menschen zueinander. }\end{array}$ & $\begin{array}{l}\text { Hauptschule: } M=3.31 \\
\text { Realschule: } M=3.53 \\
\text { Gesamtschule: } M=3.84 \\
\text { (Gymnasium } M=2.78 \text { ) } \\
\\
\text { Deutsche: } M=2.71 \\
\text { (Koreaner } M=3.06 \text {; Italiener } \\
M=3.28 ; \text { Türken } M=3.90 \text { ) }\end{array}$ & $\begin{array}{r}-2.26 \\
-3.10 \\
-3.51 \\
\\
3.38\end{array}$ & $\begin{array}{l}0.026 \\
0.002 \\
0.001 \\
0.001\end{array}$ \\
\hline $\begin{array}{l}\text { In einer Ehe muss man viele persönliche } \\
\text { Wünsche zurückstellen. }\end{array}$ & $\begin{array}{l}\text { Christen: } \mathrm{M}=2.85 \\
\text { (Nicht-Christen } \mathrm{M}=3.33 \text { ) }\end{array}$ & 1.99 & 0.049 \\
\hline $\begin{array}{l}\text { Die Ehe ist ein ganz normaler Teil des Le- } \\
\text { bens, der zum Erwachsenenleben dazuge- } \\
\text { hört. }\end{array}$ & $\begin{array}{l}\text { Deutsche: } \mathrm{M}=2.00 \\
\text { (Italiener } \mathrm{M}=2.38 ; \text { Koreaner } \\
\mathrm{M}=2.72 ; \text { Türken } \mathrm{M}=3.00 \text { ) } \\
\text { weiblich: } \mathrm{M}=3.18 \\
\text { (männlich } \mathrm{M}=2.83 \text { ) }\end{array}$ & $\begin{array}{r}3.09 \\
-2.80\end{array}$ & 0.006 \\
\hline
\end{tabular}

Die beeinflussenden Faktoren wurden von der Regressionsanalyse als signifikant ausgewiesen; die in Klammern gesetzten Daten sind zur Information ergänzt ( $1=$ sehr hohe Ablehnung bis $5=$ sehr hohe Zustimmung).

Zur Perspektive der Eltern bezogen auf eine Ehe der Befragten finden sich die in Tabelle 4 aufgeführten Prädiktoren; bedeutsam waren hier die ethnische Zugehörigkeit, die Religion, die Schulbildung und das Geschlecht. Zur „Aufgabenverteilung in der Ehe" und in der wahrgenommenen Übereinstimmung zwischen den Elternerwartungen und den eigenen Vorstellungen finden sich keine signifikanten Unterschiede zwischen den Untersuchungsgruppen. Zur Aufgabenverteilung im Elternhaus befragt, war die meistgenannte Aufgabe des Vaters „Geld verdienen“; die koreanischen Probanden hatten dies als gemeinsame Aufgaben der Eltern erfahren. In den anderen Gruppen waren die zentralen Aufgaben der Mutter „Haushaltsführung“ und „Kindererziehung“, wobei letzteres auch gemeinsame Aufgabe der Eltern war. 
Tabelle 4: $\quad$ Signifikante Ergebnisse der Regressionsanalyse für den Bereich „Perspektive der Eltern“ in Studie $1(\mathrm{~N}=115)$

\begin{tabular}{|c|c|c|c|}
\hline Item & Beeinflussende Faktoren & $\mathrm{T}$ & $\mathrm{p}$ \\
\hline $\begin{array}{l}\text { Es ist meinen Eltern wichtig, dass ich einen } \\
\text { Partner aus unserer Kultur heirate. }\end{array}$ & $\begin{array}{l}\text { Deutsche: } M=2.03 \\
\text { Italiener: } M=2.93 \\
\text { (Koreaner } M=3.44 \text {; Türken } \\
M=3.86 \text { ) }\end{array}$ & $\begin{array}{l}5.19 \\
2.35\end{array}$ & $\begin{array}{r}<0.001 \\
0.021\end{array}$ \\
\hline Meine Eltern erwarten Enkelkinder. & $\begin{array}{l}\text { Deutsche: } M=3.29 \\
\text { (Koreaner } M=4.38 \text {; Türken } \\
M=4.38 \text {; Italiener } M=4.41 \text { ) }\end{array}$ & 4.82 & $<0.001$ \\
\hline $\begin{array}{l}\text { Meine Eltern erwarten von mir, dass ich } \\
\text { heirate. }\end{array}$ & $\begin{array}{l}\text { Italiener: } M=3.17 \\
\text { Türken: } M=3.18 \\
\text { Koreaner: } M=4.03 \\
\text { (Deutsche } M=1.71 \text { ) }\end{array}$ & $\begin{array}{l}-4.50 \\
-4.50 \\
-5.92\end{array}$ & $\begin{array}{l}<0.001 \\
<0.001 \\
<0.001\end{array}$ \\
\hline $\begin{array}{l}\text { Meine Eltern erwarten von mir, dass ich } \\
\text { meine persönlichen Interessen hinter die } \\
\text { Interessen der Ehe/meines Partners stelle. }\end{array}$ & $\begin{array}{l}\text { Christen: } \mathrm{M}=2.14 \\
\text { (Nicht-Christen } \mathrm{M}=2.94 \text { ) } \\
\text { Deutsche: } \mathrm{M}=1.75 \\
\text { (Koreaner } \mathrm{M}=2.28 \text {; Italiener } \\
\mathrm{M}=2.55 ; \text { Türken } \mathrm{M}=2.97 \text { ) } \\
\text { weiblich: } \mathrm{M}=2.10 \\
\text { (männlich } \mathrm{M}=2.71 \text { ) }\end{array}$ & $\begin{array}{l}2.35 \\
2.86 \\
2.24\end{array}$ & $\begin{array}{l}0.021 \\
0.005 \\
0.027\end{array}$ \\
\hline $\begin{array}{l}\text { Für meine Eltern ist meine Heirat ein Zei- } \\
\text { chen meiner Reife. }\end{array}$ & $\begin{array}{l}\text { Italiener: } M=2.72 \\
\text { Türken: } M=2.72 \\
\text { Koreaner: } M=3.07 \\
\text { (Deutsche } M=1.86 \text { ) } \\
\text { Hauptschule: } M=2.39 \\
\text { (Gesamtschule } M=2.42 \text {; Gymna- } \\
\text { sium } M=2.71 \text {; Realschule } \\
M=2.95 \text { ) }\end{array}$ & $\begin{array}{l}-4.50 \\
-4.50 \\
-5.92 \\
\\
2.29\end{array}$ & $\begin{array}{r}<0.001 \\
<0.001 \\
<0.001 \\
\\
\\
\end{array}$ \\
\hline $\begin{array}{l}\text { Meine Eltern bestehen darauf, bei der Part- } \\
\text { nerwahl mitzubestimmen. }\end{array}$ & $\begin{array}{l}\text { Christen: } \mathrm{M}=1.59 \\
\text { (Nicht-Christen } \mathrm{M}=2.00 \text { ) } \\
\text { Koreaner: } \mathrm{M}=2.34 \\
\text { (Deutsche } \mathrm{M}=1.11 \text {; Italiener } \\
\mathrm{M}=1.34 ; \text { Türken } \mathrm{M}=2.07 \text { ) }\end{array}$ & $\begin{array}{r}2.90 \\
-3.71\end{array}$ & $\begin{array}{r}0.005 \\
<0.001\end{array}$ \\
\hline
\end{tabular}

Die beeinflussenden Faktoren wurden von der Regressionsanalyse als signifikant ausgewiesen; die in Klammern gesetzten Daten sind zur Information ergänzt ( $1=$ sehr hohe Ablehnung bis $5=$ sehr hohe Zustimmung).

\subsubsection{Gründung der eigenen Familie}

Nach den Voraussetzungen zur Gründung einer eigenen Familie aus der Sicht der Probanden wurde unter den drei häufigsten Antworten von allen Gruppen „finanzielle Unabhängigkeit" genannt. Die deutschen und italienischen Befragten gaben zusätzlich „Liebe“ und „emotionale Zufriedenheit“ an, die koreanischen den „,richtigen Partner“ und „Entschlossenheit/Reife“ und die türkischen Probanden wollten die Voraussetzung „emotionale Zufriedenheit“ und „den richtigen Partner“ erfüllt wissen.

Als erwünschte Familienkonstellation gaben die italienischen und türkischen jungen Erwachsenen fast ausschließlich „Mann und Frau, als Ehepaar, mit Kin- 
dern“ an, wohingegen die Deutschen und Koreaner sich auch andere Familienzusammenstellungen (unverheiratet, ohne Kinder oder allein erziehender Elternteil) für die eigene Familiengestaltung vorstellen konnten.

„Kinder sind gut zur Festigung der Ehe.“ $(\mathrm{F}(3,111)=7.57, \mathrm{p}<0.001)$ wurde von den deutschen Probanden $(\mathrm{M}=2.36)(\mathrm{T}=2.87, \mathrm{p}=0.005)$ abgelehnt, während die ausländischen Probanden weder zustimmten noch widersprachen. Auch Frauen $(\mathrm{M}=2.67)$ lehnten die Aussage eher $\mathrm{ab}(\mathrm{T}=3.10, \mathrm{p}=0.002)$ als Männer $(\mathrm{M}=3.38)$, die dieser Sichtweise eher zustimmten. Bezüglich des Items „Kinder sind eine finanzielle Belastung." $(\mathrm{F}(3,110)=6.20, \mathrm{p}=0.001)$ fand sich bei den Italienern $(\mathrm{M}=2.14)$ die stärkste Ablehnung. Das Item „Kinder schaffen Probleme mit Nachbarn, auf Reisen und in der Öffentlichkeit." $(\mathrm{F}(2,112)=10.34, \mathrm{p}<0.001)$ lehnten Christen $(\mathrm{M}=1.85)(\mathrm{T}=3.96, \mathrm{p}<0.001)$ deutlich stärker ab als NichtChristen $(\mathrm{M}=2.75)$.

Im Bereich „Kindererziehung“ wies die multivariate Überprüfung Prädiktoren aus, die die Ethnie und die Schulbildung betreffen. Hauptschüler $(M=3.33)$ stimmten der Aussage „Für die Entwicklung des Kindes ist es schlecht, wenn die Mutter berufstätig ist.“ $(\mathrm{F}(1,113)=7.35, \mathrm{p}=0.008)$ eher zu als Absolventen anderer Schulformen. Das Item „Der Vater sollte eher das Vorbild für den Sohn sein als die Mutter" $(\mathrm{F}(2,112)=3.23, \mathrm{p}=0.043)$ lehnten die Koreaner $(\mathrm{M}=2.76)$ weniger stark ab als die anderen Untersuchungsteilnehmer. Nur Deutsche $(\mathrm{M}=2.25)$ und Italiener $(\mathrm{M}=2.24)$ widersprachen der Aussage ,In den ersten Lebensjahren braucht das Kind mehr die Nähe zur Mutter als zum Vater“ $(\mathrm{F}(2,112)=11.33$, $\mathrm{p}<$ 0.001). In den Erwartungen, die die Befragten an ihre eigenen Kinder hatten, unterschieden sich die Untersuchungsgruppen nicht voneinander; „Glück der Kinder“, „Erfolg in der Schule“ und „,das Zurechtkommen mit anderen Kindern“ wurden am häufigsten angegeben.

Insgesamt setzte sich bei den 36 Regressionsanalysen 20 Mal „Ethnie“ als einflussreichster Faktor durch, 13 Mal als einzelne Variable und sieben Mal in Kombination mit „Geschlecht“, „Schulbesuch“ oder „Religion“.

\subsubsection{Profilbildung}

Die explorative Faktorenanalyse ergab sechs Faktoren, mit deren Hilfe genauere Profile der einzelnen ethnischen Gruppen spezifiziert werden sollten:

- „Innere Werte“ bezüglich Partnerschaft und Ehe: Toleranz, Ehrlichkeit, Treue, Kompromissbereitschaft, Unterstützung usw. als Voraussetzungen für Partnerschaft und Ehe;

- „Äußere Kriterien“ bezüglich Partnerschaft und Ehe: gleiche Religion, gute finanzielle Situation, gesellschaftliches Ansehen und Familienhintergründe des anderen als Voraussetzungen für eine gute Partnerschaft;

- „Traditionelle Sichtweise“ bezüglich Partnerschaft und Kindern: traditionelle Rollenverteilung in der Partnerschaft, der Ehe und bei der Erziehung der Kinder; 
- „Kinder und Ehe als Einschränkung“ in der Selbstverwirklichung: z.B. „In einer Ehe muss man viele persönliche Wünsche zurückstellen.“, „Kinder lassen wenig Zeit für eigene Interessen.“, „Sie sind eine finanzielle Belastung“;

- „Emotionale Bereicherung durch Kinder“: Kinder machen Freude, bereichern das Leben;

- „Elterneinfluss“: Für die Eltern ist die Ehe ein Zeichen der Reife des Kindes, Eltern wollen bei der Partnerwahl mitbestimmen; sie erwarten Enkelkinder, usw.

Nach der Varianzanalyse unterscheiden sich die Probanden in der Bewertung bei vier von sechs Faktoren signifikant voneinander. Die Mittelwerte der Untersuchungsgruppen für diese Faktoren gibt Abbildung 1 wieder.

Abbildung 1: Mittelwerte für die Bereiche „Innere Werte“, „Traditionelle Sichtweise“, „Ehe und Kinder als Einschränkung“ und „Elterneinfluss“ in Studie $1(\mathrm{~N}=115)$.
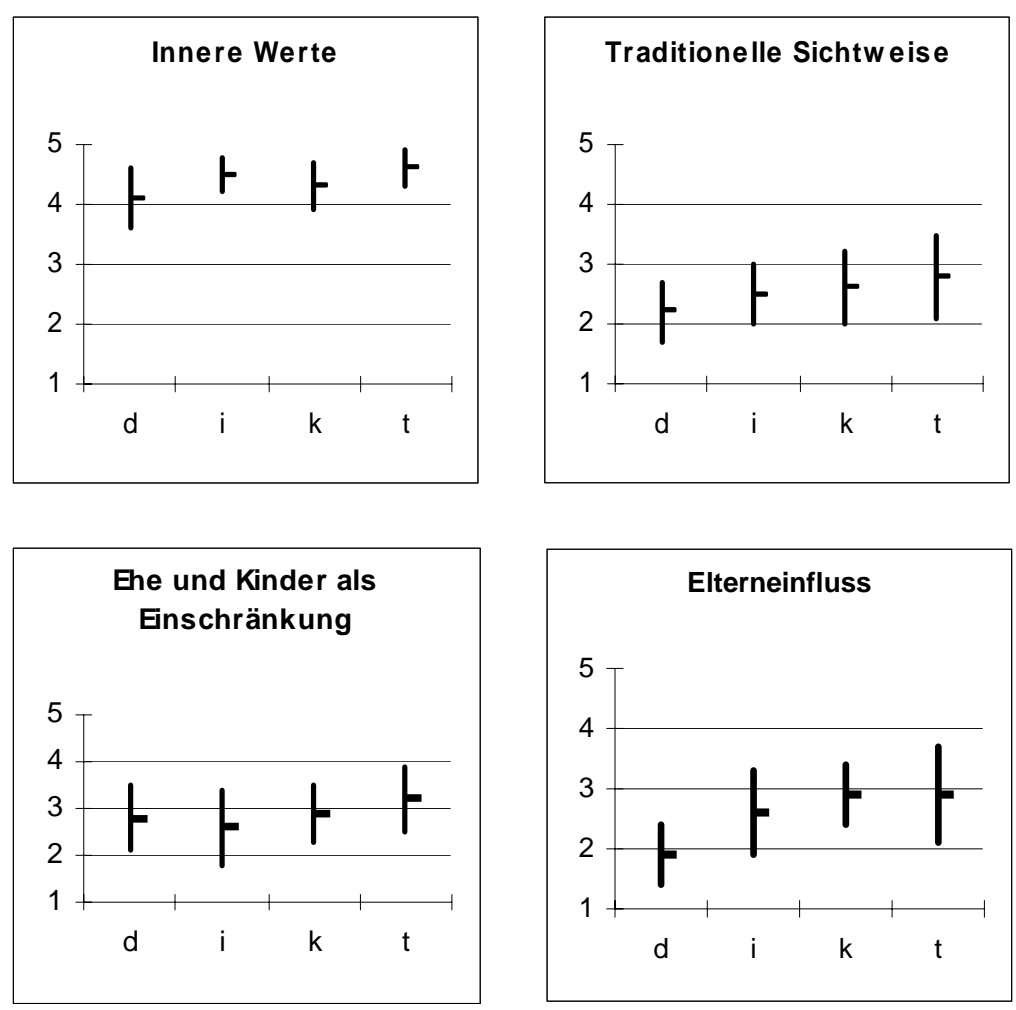

( 1 = sehr hohe Ablehnung bis $5=$ sehr hohe Zustimmung; $d=$ Deutsche, $i=$ Italiener, $k=$ Koreaner und $\mathrm{t}=$ Türken) 
Der Post-hoc-Test (nach Scheffé) lässt bei „Innere Werte“ $(\mathrm{F}(3,111)=6.96$, p < 0.001) zwei mögliche Untergruppenbildungen zu. Zum einen können die deutschen in eine und alle ausländischen Probanden in eine zweite Gruppe gebracht werden; zum anderen können die italienischen und türkischen Untersuchungsteilnehmer eine und die deutschen und koreanischen die andere Gruppe bilden. Beim Faktor „Traditionelle Sichtweisen“ $(\mathrm{F}(3,111)=4.59, \mathrm{p}=0.005)$ finden sich entweder die deutschen oder die türkischen jungen Erwachsenen in einer separaten Gruppe. Auch bei der Bewertung von „Kinder und Ehe als Einschränkung“ $(\mathrm{F}(3,111)=4.15, \mathrm{p}=0.008)$ können die türkischen Befragten eine Gruppe für sich bilden, ebenso die Gruppe der italienischen Befragten. Bezogen auf den „Elterneinfluss“ $(\mathrm{F}(3,111)=16.58, \mathrm{p}<0.001)$ unterscheiden sich die ausländischen Teilnehmer deutlich in ihrer Bewertung von den deutschen. Geschlechtsspezifische Unterschiede ließen sich nicht finden.

\section{Studie 2: Familienvorstellungen von jungen Erwachsenen mit Abitur ${ }^{1}$}

In Studie 1 erwies sich neben der kulturellen Zugehörigkeit auch die Bildungsvariable als bedeutsam. Aufgrund der angestrebten Repräsentativität in den Bildungsvoraussetzungen wurden in den Gruppen der italienischen und türkischen Probanden größtenteils junge Erwachsene befragt, die die Haupt- oder Realschule besucht hatten, bei den Koreanern dagegen fast ausschließlich ehemalige Gymnasiasten. Die Geschlechtszugehörigkeit der Befragten erwies sich in den Regressionsanalysen ebenfalls als bedeutsam. Diese Ergebnisse legen die Vermutung nahe, dass sich mit einer weiterführenden Bildung im deutschen Schulsystem die Vorstellungen vom geplanten Leben als Familie verändern. Es kann angenommen werden, dass gerade junge Migrantinnen eine eher selbstbestimmte und weniger an Traditionen ausgerichtete Perspektive für ihre Zukunft realisieren möchten, wenn sie eine weiterführende Schule besuchten und damit größere Berufschancen erlangen konnten. Daher wurde eine ergänzende Stichprobe befragt, die es erlaubt, den Einfluss der Variablen Gymnasialbildung und Geschlecht systematisch zu untersuchen.

\subsection{Methode}

Weitere 100 Personen wurden befragt. Durch die Ausweitung der Stichprobe lagen nun Gruppen mit jeweils 20 Gymnasialabsolventinnen und jeweils 15 Männern mit Abitur aus allen vier Ethnien vor. Die Datenauswertung erfolgte zunächst wie in Studie 1. Um Effekte der Bildungsvariablen zu überprüfen, wurden die Daten

1 Gefördert mit Mitteln für Frauenforschung der Universität Bonn. Wir danken Dipl.Psych. Miriam Traunfelder insbesondere für die Datenerhebung bei den italienischen Abiturienten. 
der Gymnasiasten mit denen der Abgänger der anderen Schultypen aus der ersten Studie verglichen.

\subsection{Ergebnisse}

Bei Konstanthalten der Bildungsvariablen nivellierten sich zahlreiche der zuvor gefundenen Ergebnisse, die bei der schulisch repräsentativen Stichprobe in Studie 1 gefunden worden waren. Andere Aspekte der Familienvorstellungen rückten im interethnischen Vergleich in den Vordergrund.

\subsubsection{Vorstellungen zur Partnerschaft}

Die Italiener mit Abitur $(\mathrm{M}=4.43)$ befürworteten zwar am stärksten eine Partnerschaft mit jemandem aus dem Heimatland der Eltern $(\mathrm{F}(3,101)=14.26$, p < $0.001)$. Gleichzeitig sprachen sie $(\mathrm{M}=4.00)$ sich aber gemeinsam mit den Koreanern $(\mathrm{M}=3.83)$ stärker für eine Partnerschaft mit einem deutschen Partner aus als die türkischen Probanden $(\mathrm{M}=3.03)(\mathrm{F}(3,101)=5.98, \mathrm{p}=0.001)$ und stärker als die Italiener ohne Abitur $(\mathrm{M}=2.93)(\mathrm{t}(59)=3.37, \mathrm{p}=0.001)$. Die Items, die die Selbständigkeit und Gleichberechtigung der Partner enthielten, wurden von allen Männer und Frauen mit Gymnasialbildung in ähnlicher Weise befürwortend beantwortet.

\subsubsection{Vorstellungen zur Ehe}

Zum Themenkomplex „Bedeutung und Wert von Ehe“ war bei der Variablen „Die Ehe ist ein Symbol ewiger Treue." $(\mathrm{F}(2,137)=9.13, \mathrm{p}<0.001)$ die Zustimmung der Deutschen $(\mathrm{M}=3.26)$ wie in Studie 1 deutlich verhaltener als bei den ausländischen Befragten. In der Bewertung der Aussage „Die Ehe verbessert die Beziehung der beiden Menschen zueinander." $(\mathrm{F}(1,138)=5.25, \mathrm{p}=0.023)$ ist die Gewichtung hier eine andere: Sowohl Italiener $(\mathrm{M}=2.86)$ als auch Deutsche $(\mathrm{M}=$ 2.66) äußerten sich eher ablehnend. Die Deutschen $(M=4.26)$ stimmten dem Item „Die Ehe hält heute selten, bis dass der Tod sie scheidet" $(\mathrm{F}(2,136)=4.52, \mathrm{p}=$ 0.012) stärker zu als die anderen Gruppen.

Zum Themenkomplex „Aufgabenverteilung in der Ehe“ ergaben sich - anders als in Studie 1 - nun einige signifikante Unterschiede zwischen den Untersuchungsgruppen. Sie sind in Tabelle 5 wiedergegeben. Bemerkenswert ist, dass bei jedem Item die Frauen signifikant anders antworteten als die Männer. Im Vergleich der Frauen untereinander zeigt sich, dass die deutschen Frauen mit Abitur die Aussage „Hausfrau und Mutter zu sein, kann eine Frau ausfüllen.“ (M = 3.15) eher bejahten, während die türkischen Frauen mit Abitur diese Aussage vehement ablehnten $(\mathrm{M}=1.85)$. In Studie 1 war die Ablehnung dieses Items auf Seiten der türkischen Untersuchungsteilnehmer ohne Abitur überzufällig moderater $(\mathrm{N}=27$; $\mathrm{M}=2.75)(\mathrm{t}(43)=-2.29, \mathrm{p}=0.027)$. Dennoch sahen die türkischen Frauen mit Abitur eine Hochzeit durchaus als normalen Teil des Erwachsenlebens an. 
Tabelle 5: $\quad$ Signifikante Ergebnisse der Regressionsanalyse für den Bereich „Aufgabenteilung in der Ehe“ aus Studie $2(\mathrm{~N}=140)$

\begin{tabular}{|c|c|c|c|}
\hline Item & Beeinflussende Faktoren & $\mathrm{T}$ & $\mathrm{p}$ \\
\hline $\begin{array}{l}\text { Mann und Frau sollten in der Ehe gleich- } \\
\text { berechtigt sein und gemeinsam alle Ent- } \\
\text { scheidungen treffen. }\end{array}$ & $\begin{array}{l}\text { Deutsche: } M=4.97 \\
\text { Italiener: } M=4.83 \\
\text { (Türken } M=4.43 \text {; Koreaner } \\
M=4.51 \text { ) } \\
\text { weiblich: } M=4.80 \\
\text { (männlich } M=4.53 \text { ) }\end{array}$ & $\begin{array}{l}-3.67 \\
-2.62 \\
\\
-2.37\end{array}$ & 0.019 \\
\hline $\begin{array}{l}\text { Die berufliche Karriere des Mannes ist } \\
\text { wichtiger als die der Frau. }\end{array}$ & $\begin{array}{l}\text { Deutsche: } M=1.43 \\
\text { Italiener: } M=1.49 \\
\text { (Türken } M=1.97 ; \text { Koreaner } \\
M=2.17 \text { ) } \\
\text { weiblich: } M=1.55 \\
\text { (männlich } M=2.05 \text { ) }\end{array}$ & $\begin{array}{l}2.83 \\
2.58 \\
\\
2.67\end{array}$ & $\begin{array}{l}0.005 \\
0.011 \\
0.008\end{array}$ \\
\hline $\begin{array}{l}\text { Bei Meinungsverschiedenheiten zwischen } \\
\text { Mann und Frau soll in jedem Fall der Mann } \\
\text { entscheiden. }\end{array}$ & $\begin{array}{l}\text { Türken: } M=1.74 \\
\text { (Deutsche } M=1.06 \text {; Italiener } \\
M=1.32 ; \text { Koreaner } M=1.46 \text { ) } \\
\text { weiblich: } M=1.06 \\
\text { (männlich } M=1.83 \text { ) }\end{array}$ & $\begin{array}{r}-2.92 \\
5.50\end{array}$ & $\begin{array}{r}0.004 \\
<0.001\end{array}$ \\
\hline $\begin{array}{l}\text { Bei großen finanziellen Entscheidungen } \\
\text { hat der Mann das letzte Wort. }\end{array}$ & $\begin{array}{l}\text { Deutsche: } M=1.11 \\
\text { Türken: } M=2.03 \\
\text { (Italiener } M=1.43 \text {; Koreaner } \\
M=1.57 \text { ) } \\
\text { weiblich: } M=1.15 \\
\text { (männlich } M=2.05 \text { ) }\end{array}$ & 6.47 & $\begin{array}{r}0.035 \\
0.002 \\
<0.001\end{array}$ \\
\hline $\begin{array}{l}\text { Den Haushalt hat in jedem Fall die Frau } \\
\text { alleine zu führen. }\end{array}$ & $\begin{array}{l}\text { Deutsche: } M=1.09 \\
\text { (Italiener } M=1.49 ; \text { Koreaner } \\
M=1.63 ; \text { Türken } M=1.83 \text { ) } \\
\text { weiblich: } M=1.16 \\
\text { (männlich } M=1.98 \text { ) }\end{array}$ & 5.13 & $\begin{array}{r}0.003 \\
<0.001\end{array}$ \\
\hline
\end{tabular}

Die beeinflussenden Faktoren wurden von der Regressionsanalyse als signifikant ausgewiesen; die in Klammern gesetzten Daten sind zur Information ergänzt ( 1 = sehr hohe Ablehnung bis $5=$ sehr hohe Zustimmung).

Für den Bereich „Perspektive der Eltern“ unterschieden sich die ethnischen Gruppen bei denselben Items wie in Studie 1. Zusätzlich stimmten die Deutschen ( $\mathrm{M}=$ 4.06), Italiener $(M=3.89)$ und Türken $(M=3.77)$ in Studie 2 dem Item ,Es ist meinen Eltern lieber, ich lasse mich wieder scheiden, als dass ich unglücklich in meiner Ehe bin." stärker zu als die Koreaner $(\mathrm{M}=3.12)(\mathrm{F}(2,136)=7.23, \mathrm{p}=$ 0.001). Insgesamt veränderten sich die Bewertungen der einzelnen Gruppen im Vergleich mit den Werten aus Studie I nicht; somit kann an dieser Stelle auf eine detaillierte Darstellung verzichtet werden. Allerdings ist anzumerken, dass bei der multivariaten Überprüfung der Items häufiger als in Studie 1 Frauen als Prädiktor aufgezeigt wurden. Außerdem zeigten die deutschen und italienischen Probanden mit Abitur eine höhere Übereinstimmung mit den wenig traditionell geprägten Erwartungen ihrer Eltern $(F(3,135)=4.70, p=0.004)$, während die koreanischen und 
türkischen jungen Erwachsenen die Erwartungen ihrer Eltern an ein den Traditionen entsprechendes Verhalten stärker wahrnahmen und zugleich weniger mit diesen übereinstimmten.

\subsubsection{Gründung der eigenen Familie}

Ein Drittel der Italiener mit Abitur konnte sich als eigene Familienkonstellation vorstellen, unverheiratet mit Kindern zusammenzuleben. Dahingegen blieb bei den türkischen Probanden die gewünschte Familienkonstellation aus einem verheirateten Paar mit Kindern bestehen. Bezogen auf die „Kindererziehung“ blieb das Item „In den ersten Lebensjahren braucht das Kind mehr die Nähe zur Mutter als zum Vater." $(\mathrm{F}(2,137)=10.69, \mathrm{p}<0.001)$ erhalten, dem die Deutschen $(\mathrm{M}=2.17)$ und Italiener $(\mathrm{M}=2.83)$ unverändert widersprachen. Bei den Items „Die Mutter sollte eher das Vorbild sein für die Tochter als der Vater" $(\mathrm{F}(2,136)=6.31, \mathrm{p}=0.002)$ und „Männer haben einfach nicht soviel Gespür für die Bedürfnisse von Kinder“ $(\mathrm{F}(3,136)=6,81, \mathrm{p}<0.001)$ zeigte sich eine noch ablehnendere Einstellung der deutschen $(\mathrm{M}=1.89$ bzw. $\mathrm{M}=1.74)$ und italienischen $(\mathrm{M}=2.21$ bzw. $\mathrm{M}=1.94)$ Probanden. Zusätzlich wurden bei den beiden letztgenannten Items Frauen als signifikante Prädiktoren ausgewiesen.

Insgesamt setzten sich bei den Regressionsanalysen 18 Mal „Ethnie“ und 17 Mal „Ethnie“ in Kombination mit „Geschlecht“ als einflussreichste Faktoren durch.

\subsubsection{Profilbildung}

Nach der Varianzanalyse unterscheiden sich die Probanden mit Gymnasialbildung in der Bewertung der sechs Faktoren, wobei in drei Fällen das Geschlecht „weiblich“ als sehr bzw. hochsignifikanter Prädiktor ausgewiesen wird. Daher und weil auch nach den bisherigen Berechnungen die Frauen viel häufiger als in Studie 1 anders als die Männer urteilten, scheint es lohnend, die Teilstichprobe der Frauen mit Abitur genauer zu beleuchten. Hier nivellieren sich die unterschiedlichen Bewertungen zu den Faktoren „Ehe und Kinder als Einschränkung“ und „Innere Werte“. Die Mittelwerte der Frauenstichprobe zu den vier Faktoren, die zu signifikanten Unterschieden zwischen den Ethnien führten, sind in Abbildung 2 wiedergegeben.

Auffallend sind die Gruppenbildungen: deutsch/italienisch und koreanisch/türkisch. Für den Bereich „Traditionelle Sichtweise“ $(F(4,135)=12.62, p<0.001)$ er-gab sich bei den deutschen und italienischen Frauen eine hohe Ablehnung. Auch die Wichtigkeit der „Äußeren Kriterien“ $(F(3,130)=8.23, \mathrm{p}<0.001)$ für eine Partnerwahl wurde von den Deutschen und Italienerinnen signifikant stärker abgelehnt als von den anderen beiden Gruppen. Dem Faktor „Emotionale Bereicherung durch Kinder" $(\mathrm{F}(1,138)=12.78, \mathrm{p}<0.001)$ stimmten die deutschen und italienischen Probandinnen etwas deutlicher zu als die koreanischen und türkischen. Diese wiederum erlebten den „Elterneinfluss“ $(F(2,137)=35.70, p<0.001)$ stärker.

Obwohl sich in beiden Untersuchungen sowohl signifikante geschlechtsspezifische als auch nationalitätenspezifische Unterschiede für die sechs durch die Fakto- 
renanalyse ermittelten Faktoren ergaben, ließen sich varianzanalytisch keine Items finden, bei denen gleichzeitig das Geschlecht und eine bestimmte Ethnie als Einflussfaktoren wirksam waren.

Abbildung 2: Mittelwerte für die Bereiche „Traditionelle Sichtweise“, „Äußere Kriterien“, „Emotionale Bereicherung durch Kinder“ und „Elterneinfluss“ der Frauen in Studie $2(\mathrm{~N}=80)$.
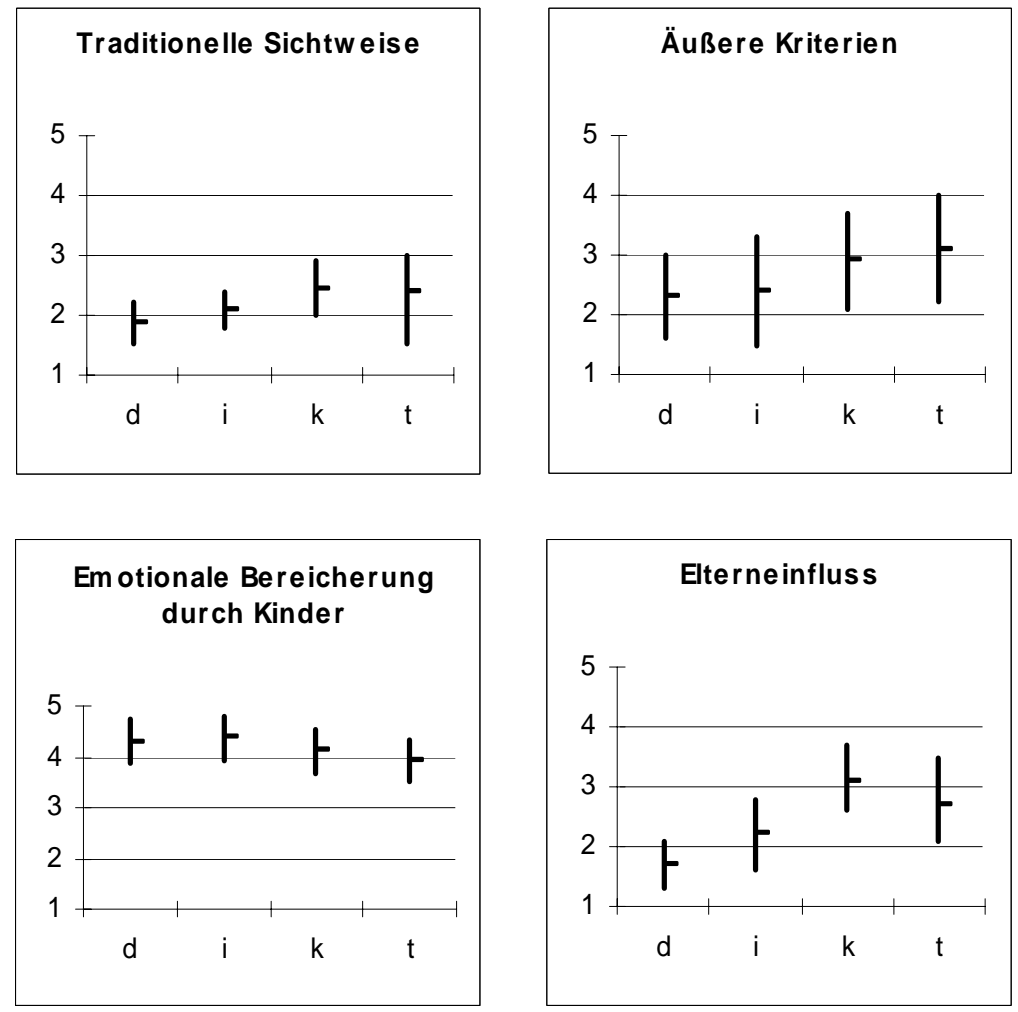

( 1 = sehr hohe Ablehnung bis $5=$ sehr hohe Zustimmung; $d$ = Deutsche, $\mathrm{i}=$ Italiener, $\mathrm{k}=$ Koreaner und $\mathrm{t}=$ Türken)

\section{Diskussion}

In Studie 1, in der die Stichproben repräsentativ nach dem erreichten Bildungsabschluss der jeweiligen kulturellen Gruppen in Nordrhein-Westfalen zusammengesetzt waren, gehen die Familienvorstellungen der untersuchten Ethnien zum Teil stark auseinander. Das Antwortverhalten der deutschen Probanden zu den Bereichen Partnerschaft, Ehe und Familie unterscheidet sich in verschiedenen Hinsichten von dem der anderen Probanden. So halten sie in den Bereichen „Partner- 
schaft“ und „Ehe“ wenig an traditionellen Vorstellungen fest und die Eltern scheinen kaum bestimmte Erwartungen an eine mögliche Ehe und Familiengründung ihrer Kinder zu stellen. Dies bestätigt Befunde etwa bei Peukert (1996) oder Engstler (1997). In den meisten Fällen findet sich eine Reihung der Ähnlichkeit der Antworten von den deutschen zu den koreanischen, dann zu den italienischen und schließlich den türkischen jungen Erwachsenen, die sich insgesamt in ihren Bewertungen am stärksten von den Deutschen unterscheiden.

Bei den koreanischen Probanden, deren Vorstellungen zur Gleichberechtigung der Partner und der Familiengestaltung denen der Deutschen in vielen Hinsichten entsprechen, ist die Bereitschaft zur Partnerschaft mit Deutschen hoch. Allerdings ist ihnen das Einverständnis bzw. die Mitsprache der Eltern bei der Partner- und Ehepartnerwahl wichtig und verschiedene traditionelle Rollenbilder werden befürwortet: So wird ein Kind auch als Unterstützung im Alter gesehen und es wird davon ausgegangen, dass kleinere Kinder vor allem die Zuneigung der Mutter benötigten. Dieser Einfluss traditioneller Wertvorstellungen zeigte sich auch in einer vergleichenden Untersuchung mit koreanischen Elternpaaren in Korea und deutschen Eltern in Deutschland (Quaiser-Pohl 1999).

Die Italiener werden in der Literatur häufig als die Migranten mit der geringsten kulturellen Distanz zu Deutschen beschrieben und treten in vielen Untersuchungen als diejenigen auf, welche sich am deutlichsten für eine Partnerschaft mit einem deutschen Partner aussprechen (z.B. Boos-Nünning 1998; Granato 1998; Nauck 2000; Weidacher 2000). In der vorliegenden Untersuchung ist dagegen nur eine mäßig ausgeprägte Bereitschaft zur Partnerschaft mit Deutschen festzustellen. Als gute Voraussetzung für eine gelungene Beziehung betrachten die italienischen Migranten vor allem einen ähnlichen kulturellen Hintergrund der Partner; das Einverständnis der Eltern ist ihnen weniger wichtig. In der Kindererziehung werden beide Eltern als zentrale Bezugspersonen erachtet, wobei Kinder am wenigsten unter dem Aspekt finanzieller Belastung angesehen werden. In ihren Vorstellungen zur Partnerschaft, den wahrgenommenen Erwartungen ihrer Eltern sowie dem Wert, den sie einer Ehe zumessen, zeigen sich unerwarteter Weise viele Ähnlichkeiten mit den türkischen Untersuchungsteilnehmern. Diese betonen für Partnerschaft und Ehe noch stärker als die anderen Gruppen die Bedeutung von Werten wie Ehrlichkeit, Treue, Kompromissbereitschaft und gegenseitige Unterstützung. Sie lehnen Aussagen, die Kinder und eine Ehe als Einschränkung beschreiben, weniger deutlich ab als die anderen kulturellen Gruppen. Die Religion sehen sie als gewichtigen Faktor in allen Bereichen des Lebens an (vgl. z.B. Özkara 1990; Zentrum für Türkeistudien 1994; Atabay 1998).

Allen weiblichen Probanden scheint die Religion wichtiger $\mathrm{zu}$ sein als den männlichen; allerdings verneinen alle Frauen die Aussagen, die sich deutlich für eine klassische Rollenverteilung und eine traditionelle Vorstellung von Ehe aussprechen. Die Tatsache, dass „Geschlecht“ einen Einfluss auf das Antwortverhalten der Probanden hat, deutet auf eine geschlechtsspezifische Erziehung der Probanden hin. Innerhalb der ausländischen Familien wurde und wird eine solche anscheinend stärker praktiziert als in deutschen Familien. Lajios (1998) verweist im Zusammenhang mit dem Wandel der ausländischen Familien nach der Migration auf die stärker ausgeprägte Wertschätzung der Eltern von traditionellen Werten 
und damit auch auf eine geschlechtsspezifische Erziehung. Beispiele für stark geschlechtsspezifisches Verhalten junger ausländischer Frauen lassen sich in der Literatur sowohl für Italienerinnen (vgl. z.B. Boos-Nünning 1998) als auch für Türkinnen (vgl. z.B. Ministerium für Arbeit, Gesundheit und Soziales des Landes NRW 1990) der zweiten Generation finden.

Betrachtet man die Stichprobe im Hinblick auf die Religionszugehörigkeit, so zeigt sich für die Christen eine eher moderne Einstellung zur Eheschließung, Familiengründung und Einbeziehung der Eltern. Diese Tendenz lässt sich bei Keller und Gummerum (2003) wieder finden: In individualistischen Kulturen wie den christlich geprägten sind Autonomie, (emotionale) Unabhängigkeit und das Recht auf Privatsphäre zentrale Werte, die auch in der Sozialisation betont werden. Im Unterschied dazu wird das Verhalten von Menschen in kollektivistischen Kulturen stark beeinflusst von traditionell vorgegebenen Zielen, Einstellungen und Werten. Der Einzelne erlebt sich als eingebettet in ein Netz von hierarchisch geordneten sozialen Beziehungen, in denen persönliche Ziele denen der Gruppe nachgeordnet werden (vgl. Oerter/Oerter 1995; Schönpflug 2003).

Die jungen Erwachsenen mit Gymnasialbildung sehen z.B. die Ehe nicht als Indikator zur Verbesserung einer Beziehung an, während Hauptschüler z.B. die Berufstätigkeit der Mutter eher als ungünstig für die Entwicklung des Kindes einschätzen. Die Gymnasiasten scheinen eher in der Lage zu sein, ihre individuellen Lebensentwürfe unabhängig von etwaigen traditionellen Vorstellungen ihrer Eltern zu entwickeln. Dieser Befund korrespondiert mit der Assimilationstheorie von Berry $(1992 ; 1997)$, nach der sich Individuen auch innerhalb einer ethnischen Gruppe in Bezug auf ihre Assimilationsstrategien in ihren Wertvorstellungen und ihrem aktuellen Verhalten unterscheiden. Aus Studien zum Einwanderungsverhalten verschiedener Migrantengruppen in Deutschland wurde ersichtlich, dass Assimilationsunterschiede wesentlich auf die unterschiedliche Verteilung von individuellen Ressourcen - insbesondere des Bildungsniveaus - zurückführbar sind (Nauck 2002; vgl. auch Badawia 2002).

Studie 2, in der alle Teilnehmer eine Gymnasialbildung hatten, bestätigt dies sehr deutlich. In Bezug auf die Selbständigkeit und Gleichberechtigung der Partner und eine weitgehende Unabhängigkeit der Lebensführung von den Erwartungen der Eltern lässt sich bei allen Befragten mit Abitur Zustimmung erkennen. Allerdings fällt diese bei den Deutschen und Italienern höher aus als bei den Koreanern und Türken. Differenzen zwischen den ethnischen Gruppen im Hinblick auf die Vorstellungen von Partnerschaft, Ehe und Familie sind vergleichsweise gering.

Geschlechtsspezifische Unterschiede treten bei den ehemaligen Abiturienten vor allem im Bereich der Aufgabenverteilung in der Ehe auf; die Frauen betonen noch stärker den Anspruch der Partnerin auf Berufstätigkeit, Mitsprache bei Entscheidungen und Entlastung von Haushaltspflichten. Betrachtet man nur die Frauen mit Gymnasialbildung, finden sich überwiegend Gemeinsamkeiten zwischen den ethnischen Gruppen, wobei die Vorstellungen der Italienerinnen besonders stark denen der deutschen Frauen ähneln. Traditionelle Auffassungen wie „Die Ehe ist ein ganz normaler Teil des Erwachsenenlebens." werden von ihnen gleichermaßen deutlich abgelehnt. Deutsche wie Italienerinnen sehen Kinder eher als emotionale Bereicherung als die beiden anderen Gruppen. 
Die größten Unterschiede innerhalb der Frauenstichprobe liegen im Bereich der elterlichen Erwartungen, die von den deutschen Frauen als sehr gering, von den Koreanerinnen dagegen als gegeben wahrgenommen werden. Entgegen Studie 1 bestehen häufig Übereinstimmungen in den Familienvorstellungen von Koreanerinnen und Türkinnen. Es ist zu berücksichtigen, dass das Bildungsniveau der koreanischen Teilnehmer in beiden Studien fast identisch geblieben ist, während sich die beiden Stichproben der Türkinnen im Bildungsniveau stark unterscheiden. In Studie 1 ist bei den Koreanerinnen ein klarer Bezug zu traditionellen Werten vorhanden. Bei den Türkinnen mit Gymnasialbildung haben sich die Familienvorstellungen dagegen denen der anderen ethnischen Gruppen stark angenähert; die überwiegend traditionsorientierten Auffassungen sind nun stark gemildert. Äußere Kriterien wie eine gute finanzielle Situation und das gesellschaftliche Ansehen des Partners sind den Koreanerinnen und Türkinnen mit Abitur nicht ganz so unwichtig wie den deutschen und italienischen Frauen mit allgemeiner Hochschulreife. Auffällig bleibt bei den Türkinnen nach wie vor die Betonung der Religion: Auch wenn sie die Gleichberechtigung zwischen den Partnern betonen, ist dies nicht als Widerspruch zu einer persönlichen Bedeutsamkeit der Religion in Bezug auf die Ehe und die Weitergabe dieser Werte an die eigenen Kinder zu verstehen. Noch nicht geklärt werden kann, ob die hier gefundenen Einflüsse der Bildung auf die Familienvorstellungen sich möglicherweise auch im Heimatland finden lassen.

Zusammenfassend kann festgehalten werden, dass in Studie 1 kein hohes Maß an Angleichung der jungen Ausländer mit Blick auf die Vorstellungen zu Partnerschaft, Ehe und Familie an die Lebensvorstellungen junger Deutscher gefunden werden konnte. Studie 2 zeigt, dass der biographische Faktor „Bildung“, insbesondere bei den jungen Frauen, einen entscheidenden Einfluss hat. Migrantinnen mit Gymnasialbildung unterscheiden sich nur wenig von den deutschen Gleichaltrigen. Dies wird am stärksten bei den Italienerinnen deutlich. Junge Koreanerinnen und junge Türkinnen zeigen sich noch eher den Traditionen verhaftet - die einen auf der Grundlage einer familienbezogenen asiatischen Tradition, die anderen aufgrund ihrer Bindung an den Islam. Studie 2 belegt, dass den Vertretern der jeweiligen Ethnien nur bedingt eng umrissene Familienvorstellungen zugeordnet werden können, die primär auf die deutsche Umgebung oder den Einfluss der Herkunftskultur zurückzuführen sind. In verschiedenen Variationen werden entweder eher traditionelle oder eher moderne Betrachtungsweisen von Partnerschaft, Ehe und Familie vertreten, die sich im Kulturkontakt aufgrund der eigenen ethnischen $\mathrm{Zu}$ gehörigkeit, der Definition der eigenen Geschlechterrolle und der Bildungsbiographie entwickelt haben. 


\section{Literatur}

Atabay, I. (1998). Zwischen Tradition und Assimilation. Die zweite Generation türkischer Migranten in der Bundesrepublik. München: Lambertus Verlag.

Badawia, T. (2002). „Der dritte Stuhl“. Eine Grounded Theory-Studie zum kreativen Umgang bildungserfolgreicher Immigrantenjugendlicher mit kultureller Distanz. Frankfurt am Main: IKO-Verlag.

Berry, J.W. (1992). Acculturation and adoption in a new society. International Migration, 30, p. 70-76.

Berry, J.W. (1997): Immigration, acculturation, and adaptation. Applied Psychology: An International Review, 46, p. 5-34.

Boos-Nünning, U. (1998). Mädchen und junge Frauen italienischer Herkunft: Soziale und berufliche Situation. In: R. Alborino/K. Pölzl (Hrsg.). Italiener in Deutschland. Teilnahme oder Ausgrenzung (S. 94-109). Freiburg/Breisgau: Lambertus Verlag.

Boos-Nünning, U./Karakaşoğlu, Y. (2005). Viele Welten leben. Zur Lebenssituation von Mädchen und jungen Frauen mit Migrantenhintergrund. Münster: Waxmann Verlag.

Böttcher, A. (1998). Wege in die Elternschaft. Familienentwicklungen im Kulturvergleich. St. Augustin: Gardez Verlag.

Bratic, L./Viehböck, E. (1994). Die zweite Generation. Migrantenjugendliche im deutschsprachigen Raum. Innsbruck: Studienverlag.

Bundesministerium für Familie, Senioren, Frauen und Jugend (1997). Familien ausländischer Herkunft. Berlin.

Bundesministerium für Familie, Senioren, Frauen und Jugend (2000). Familien ausländischer Herkunft in Deutschland. Leistungen, Belastungen, Herausforderungen. Sechster Familienbericht. Berlin.

Bürkner, H.J. (1998). Jugendliche Arbeitsmigranten in Deutschland. Perspektiven am Arbeitsplatz, in Schule und Familie. Geographische Rundschau, 49 (7-8), S. 418-422.

Deutsche Shell (Hrsg.) (2000). Jugend 2000. 13. Shell Jugendstudie. Opladen: Leske + Budrich.

Engstler, H. (1997). Die Familie im Spiegel der amtlichen Statistik. Bonn: Bundesministerium für Familie, Senioren, Frauen und Jugend.

Fritzsche, I./Münchmeier, R. (2000). Mädchen und Jungen. In: Deutsche Shell (Hrsg.). Jugend 2000. 13. Shell Jugendstudie (S. 343-348). Opladen: Leske + Budrich.

Granato, M. (1998). Italienische Jugendliche in der Bundesrepublik: Leben in der Migration zwischen Integration und Ausgrenzung. In: R. Alborino/K. Pölzl (Hrsg.). Italiener in Deutschland. Teilnahme oder Ausgrenzung (S. 127-146). Freiburg/Breisgau: Lambertus Verlag.

Gültekin, N. (2003). Bildung, Autonomie, Tradition und Migration. Doppelperspektivität biographischer Prozesse junger Frauen aus der Türkei. Bonn: Leske + Budrich.

Horn, R. (2003). Probleme des interkulturellen Einsatzes von Fragebogen. Landau: Verlag Empirische Pädagogik.

Keller, M./Gummerum, M. (2003). Freundschaft und Verwandtschaft - Beziehungsvorstellungen im Entwicklungsverlauf und im Kulturvergleich. Sozialer Sinn, 1, S. 95-121.

Krampen, G./Reichle, B. (2002). Frühes Erwachsenenalter. In: R. Oerter/L. Montada (Hrsg.). Entwicklungspsychologie (S. 319-350). Weinheim: Psychologie Verlags Union.

Lajios, K. (1998). Die allgemeine Situation ausländischer Familien in der Bundesrepublik Deutschland. In: K. Lajios. Die ausländische Familie (S. 13-23). Opladen: Leske + Budrich.

Lee, J.-S. (1991). Koreanischer Alltag in Deutschland. Münster: Waxmann Verlag GmbH. Loriedo, C. (1996). Familie und Familientherapie in Italien. Familiendynamik, 21, S. 51-59. 
Merkens, H. (1997). Familiale Erziehung und Sozialisation türkischer Kinder in Deutschland. In: H. Merkens/F. Schmidt (Hrsg.). Sozialisation und Erziehung ausländischer Familien in Deutschland (S. 1-100). Hohengehren: Schneider Verlag.

Merkens, H./Schmidt, F. (Hrsg.) (1997). Sozialisation und Erziehung ausländischer Familien in Deutschland. Hohengehren: Schneider Verlag.

Ministerium für Arbeit, Gesundheit und Soziales des Landes NRW (1990): Türkische Mädchen und Freizeit. Düsseldorf.

Mitteilung der Beauftragten der Bundesregierung für die Belange der Ausländer (1997). Integration oder Ausgrenzung? Zur Bildungs- und Ausbildungssituation von Jugendlichen ausländischer Herkunft. Bonn.

Münchmeier, R. (2000). Miteinander - Nebeneinander - Gegeneinander? Zum Verständnis zwischen deutschen und ausländischen Jugendlichen. In: Deutsche Shell (Hrsg.). Jugend 2000. 13. Shell Jugendstudie (S. 221-260). Opladen: Leske + Budrich.

Nauck, B. (2000). Eltern-Kind-Beziehung in Migrantenfamilien - ein Vergleich zwischen griechischen, italienischen, türkischen und vietnamesischen Familien in Deutschland. In: Sachverständigenkommission 6. Familienbericht (Hrsg.). Familien ausländischer Herkunft in Deutschland. Empirische Beiträge zur Familienentwicklung und Akkulturation (S. 347-392). Opladen: Leske + Budrich.

Nauck, B./Niephaus, Y. (2001). Intergenerative Konflikte und gesundheitliche Belastungen in Migrantenfamilien. In: P. Marschalack/ K. H. Wiedl (Hrsg.). Migration und Krankheit (S. 217-250). Osnabrück: Rasch.

Nauck, B. (2002). Dreißig Jahre Migrantenfamilien in der Bundesrepublik. Familiärer Wandel zwischen Situationsanpassung, Akkulturation, Segregation und Remigration. In: R. Nave-Herz (Hrsg.). Kontinuität und Wandel der Familien in Deutschland. Eine zeitgeschichtliche Analyse (S. 315-339). Stuttgart: Lucius \& Lucius.

Oerter, R./Oerter, R. (1995). Zur Konzeption der autonomen Identität in östlichen und westlichen Kulturen. In: G. Trommsdorff (Hrsg.). Kindheit und Jugend in verschiedenen Kulturen (S. 153-173). Weinheim: Juventa.

Özkara, S. (1990). Türkische Migranten in der Bundesrepublik Deutschland. Frankfurt am Main: Dagyeli.

Peukert, R. (1996). Familienformen im sozialen Wandel. Opladen: Leske + Budrich.

Quaiser-Pohl, C. (1999). Kindbezogene Einstellungen, Rollenauffassungen und partnerschaftliche Zufriedenheit junger Eltern aus Deutschland und Südkorea. In: B. Reichle/H. Werneck (Hrsg.). Übergang zur Elternschaft. Aktuelle Studien zur Bewältigung eines unterschätzten Lebensereignisses (S. 1-15). Stuttgart: Ferdinand Enke Verlag.

Röhr-Sendlmeier, U. M. (1990a). Zweitsprachenerwerb und personale Entwicklung. Psychologie in Unterricht und Erziehung; 37, S. 163-171.

Röhr-Sendlmeier, U. M. (1990b). Social context and the acquisition of German by Turkish migrant children. Journal of Multilingual and Multicultural Development, 11, p. 377391.

Röhr-Sendlmeier, U. M. (1992). Der Schulunterricht für Migranten in Deutschland - Maßnahmen und bildungspolitische Konzepte von 1950 bis 1990. In: H. Macha/ H.-J. Roth (Hrsg.). Bildungs- und Erziehungsgeschichte im 20. Jahrhundert (S. 297-321). Frankfurt am Main: Lang.

Schönpflug, U. (2003). Migration aus kulturvergleichender psychologischer Perspektive. In: A. Thomas (Hrsg.). Kulturvergleichende Psychologie (S. 515-541). Göttingen: Hogrefe.

Schulze, E./Soja, E. M. (2003). Verschlungene Bildungspfade. Über die Bildungskarrieren von Jugendlichen mit Migrantenhintergrund. In: G. Auernheimer (Hrsg.). Schieflage im Bildungssystem. Die Benachteiligung von Migrantenkindern (S. 97-112). Opladen: Leske + Budrich.

Seifert, W. (2000). Intergenerationale Bildungs- und Erwerbsmobilität. In: Sachverständigenkommission 6. Familienbericht (Hrsg.). Familien ausländischer Herkunft in Deutsch- 
land. Empirische Beiträge zur Familienentwicklung und Akkulturation (S. 49-86). Opladen: Leske + Budrich.

Straßburger, G. (2000). Das Heiratsverhalten von Personen ausländischer Nationalität oder Herkunft in Deutschland. In: Sachverständigenkommission 6. Familienbericht (Hrsg.). Familien ausländischer Herkunft in Deutschland. Empirische Beiträge zur Familienentwicklung und Akkulturation (S. 9-48). Opladen: Leske + Budrich.

Thränhardt, D. (1998). Inklusion und Exklusion: Die Italiener in Deutschland. In: R. Alborino/K. Plözl (Hrsg.). Italiener in Deutschland. Teilhabe oder Ausgrenzung? (S. 15-46). Freiburg/Breisgau: Lambertus Verlag.

Trommsdorff, G. (2001). Eltern-Kind-Beziehungen aus kulturvergleichender Sicht. In: S. Walper/R. Pekrum (Hrsg.). Familie und Entwicklung. Aktuelle Perspektiven der Familienpsychologie (S. 36-62). Göttingen: Hogrefe.

Vetter, S. (2001). Partnerwahl und Nationalität. Heiratsbeziehungen zwischen Ausländern in der Bundesrepublik Deutschland. In: T. Klein (Hrsg). Partnerwahl und Heiratsmuster. Sozialstrukturelle Voraussetzungen der Liebe (S. 207-232). Opladen: Leske + Budrich.

Weidacher, A. (2000). Lebensformen, Partnerschaft und Familiengründung. Griechische, italienische, türkische und deutsche junge Erwachsene. In: Sachverständigenkommission 6. Familienbericht (Hrsg.). Familien ausländischer Herkunft in Deutschland. Empirische Beiträge zur Familienentwicklung und Akkulturation (S. 193-228). Opladen: Leske + Budrich.

Yoo, J.-S. (1996). Koreanische Immigranten in Deutschland. Hamburg: Verlag Dr. Kovak. Zentrum für Türkeistudien (1994). Ausländer in der Bundesrepublik Deutschland. Opladen: Leske + Budrich.

\section{Anschrift der Autorinnen}

Prof. Dr. Una M. Röhr-Sendlmeier

Jenny Yun, M.A.

Institut für Psychologie

Abt. Entwicklungspsychologie und Pädagogische Psychologie

Rheinische Friedrich-Wilhelms-Universität Bonn

Römerstr. 164

D- 53117 Bonn

Email: roehr-sendlmeier@uni-bonn.de jenny.yun@psychologie.uni-bonn.de 